Bull. austral. Math. Soc.

VoL. 43 (1991) [491-498]

\title{
ON $N$-FLAT MODULES OVER A COMMUTATIVE RING
}

\author{
DAVID E. DOBBS
}

Let $R$ be a commutative ring with unit, $T$ an $R$-module, and $n$ a positive integer. It is proved that $T$ is $n$-flat over $R$ if $B \otimes_{R} T$ is $B$-torsionfree for each $n$-generated commutative $R$-algebra $B$. The converse holds if $T$ is $n$-generated, in which case $T$ is actually flat over $R$. Several other instances of the converse are established, but it is shown that the converse fails in general, even for $R$ an integral domain, $T$ an ideal of $R$, and $n=1$.

\section{INTRODUCTION}

Let $R$ be a commutative ring with unit. Motivated by [3, Corollary 1, p.27], we say that an $R$-module $T$ is $n$-flat (over $R$ ), where $n$ is a positive integer, in case each relation $r_{1} t_{1}+\ldots+r_{n} t_{n}=0$ (with each $r_{i} \in R, t_{i} \in T$ ) is induced by suitable $f_{j} \in T(1 \leqslant j \leqslant m)$ and $r_{i j} \in R(1 \leqslant i \leqslant n ; 1 \leqslant j \leqslant m)$ satisfying $t_{i}=\sum r_{i j} f_{j}$ for each $i$ and $\sum r_{i} r_{i j}=0$ for each $j$. According to the cited result, $T$ is $R$-flat if and only if $T$ is $n$-flat for each positive integer $n$. Since characterisations of finitely generated flat modules have been of considerable interest (see [7] and the references listed there), it seems natural to ask for conditions under which $n$-flatness implies flatness. One such is given in Corollary 2.3 where it is shown, among others, that $T$ is flat if it is $n$-flat and $n$-generated.

To explain the rest of Corollary 2.3, we need to study the connection between flatness and torsionfreeness. If $T$ is $R$-flat, then $T$ is $R$-torsionfree. The converse is false; indeed, if $R$ is an integral domain such that each torsionfree $R$-module is flat, then $R$ is a Prüfer domain (see [10], [4, Theorem 4.2], [13, Theorem 4], [5, Theorem]). One fundamental difference between flatness and torsionfreeness is that flatness is a universal property. It follows that if $T$ is $R$-flat, then $B \otimes_{R} T$ is $B$-torsionfree for each commutative $R$-algebra B. Lazarus [12] has recently established the converse of this result; in particular, an $R$-module is flat if and only if it is "universally torsionfree". Moreover, it follows from the proof of [12, Theorème 3.1] that if $B \otimes_{R} T$ is $B$-torsionfree for each $(n+2)$-generated commutative $R$-algebra $B$, then $T$ is $n$-flat. Our main result, Theorem 2.2 , succeeds in replacing " $n+2$ " with $n$. In other words, if $B \otimes_{R} T$ is $B$-torsionfree for each $n$-generated commutative $R$-algebra $B$, then $T$ is $n$-flat over

Received 28 June 1990

Copyright Clearance Centre, Inc. Serial-fee code: 0004-9729/91 \$A2.00+0.00. 
$R$. If, in addition, $T$ is $n$-generated as an $R$-module, then $T$ is actually flat over $R$ : this is part of the "rest" of Corollary 2.3 that was mentioned above. Also noteworthy in Section 2 are a new torsionfree-theoretic characterisation of Prüfer domains (Corollary 2.4); and a characterisation of $n$-flatness in terms of tensor product (Proposition 2.1), extending a result established in [2].

Section 3 is devoted to studying the converse of Theorem 2.2. In other words, if $T$ is $n$-flat over $R$, must $B \otimes_{R} T$ be $B$-torsionfree for each $n$-generated commutative $R$-algebra $B$ ? As the above references indicate, such questions are classically best understood for integral domains; so, we suppose $R$ is an integral domain and $T$ a torsionfree $R$-module, typically an integral domain containing $R$ or an ideal of $R$. Then $T$ is automatically 1-flat. Moreover, for $n \geqslant 2, n$-flatness implies 2-flatness; and 2-flatness is equivalent to $L C M$-stability (in the sense of $[9,8]$ ). It is known that if $T$ is a finitely generated ideal or an overring of an integral domain $R$, then $T$ is $R$-flat if (and only if) $T$ is $L C M$-stable over $R$ (see [1], [2, Theorem 3.5], [15, Proposition 1.7]). Accordingly, in Section 3, we focus further on the case $n=1$. In a surprising number of cases (Propositions 3.1 and 3.2 and Remark 3.3(b)), it turns out that $B \otimes_{R} T$ is $B$ torsionfree for a particular 1-generated commutative $R$-algebra $B$. However, Example 3.4 provides a negative answer; namely the converse of Theorem 2.2 is false even if $R$ is an integral domain, $T$ an ideal of $R$, and $n=1$. The construction in Example 3.4 involves a pseudo-valuation domain (in the sense of [11]) and is motivated by a dichotomy involving flatness that was noted in [6, Theorem 2.3].

Our attention was first drawn to [12] in the course of trying to make sense of a "universally $L C M$-stable" concept. (Preservation of the $L C M$-stable property under polynomial base changes has received considerable attention: see [15, Corollary 3.7], [14, Theorem 5], [16, Theorem 11]). In this vein, we show in Corollary 2.5 that for each positive integer $n$, flatness is equivalent to "universally $n$-flat." This leads to the result (Remark 3.6) that 2-flatness is not a universal property.

Throughout, all rings and algebras are assumed commutative, with unit. Unadorned $\otimes$ means $\otimes_{R}$. For convenience, " $n$-generated" means "having a generating set of cardinality at most $n . "$

\section{2. n-UNIVERSALLY TORSIONFREE MODULES ARE $n$-FLAT}

The modules referred to in this section's title are studied in Theorem 2.2. First, we give a characterisation of $n$-flatness in terms of tensor product. Note that the implication (2) $\Rightarrow(1)$ in Proposition 2.1 was obtained in case $R$ is an integral domain in [2, Proposition 3.1]; and that (1) $\Rightarrow(2)$ was obtained in case $R$ is an integral domain, $T$ is an ideal of $R$, and $n=2$ as part of [2, Theorem 3.5].

Proposition 2.1. Let $R$ be a ring, $T$ an $R$-module and $n$ a positive integer. 
Then the following conditions are equivalent:

(1) The canonical homomorphism $g: I \otimes T \rightarrow T$ is a monomorphism for each $n$-generated ideal $I$ of $R$;

(2) $T$ is $n$-flat over $R$.

Proof: One may show that (2) $\Rightarrow(1)$ exactly as in the proof of $[2$, Proposition 3.1]. For the converse, assume (1). We must show that if $r_{1} t_{1}+\ldots+r_{n} t_{n}=0$ (with $\left.r_{i} \in R, t_{i} \in T\right)$, then there exist $f_{j} \in T$ and $r_{i j} \in R(1 \leqslant j \leqslant m ; 1 \leqslant i \leqslant n)$ such that $t_{i}=\sum r_{i j} f_{j}$ for each $i$ and $\sum r_{i} r_{i j}=0$ for each $j$. Put $I=\sum R r_{i}$ and consider $\xi=\sum r_{i} \otimes t_{i}$ in $I \otimes T$. Since $g(\xi)=\sum r_{i} t_{i}=0$, it follows from (1) that $\xi=0$. An application of $\left[3\right.$, Lemma 10, p.25] now produces $f_{j}, r_{i j}$ with the desired properties, to complete the proof.

We next give our main result. As explained in the introduction, it sharpens a result of Lazarus [12, Théorème 3.1].

THEOREM 2.2. Let $R$ be a ring, $T$ an $R$-module and $n$ a positive integer. Then $T$ is $n$-flat over $R$ under each of the following conditions:

(i) $B \otimes T$ is $B$-torsionfree for each $n$-generated $R$-algebra $B$;

(ii) $B \otimes T$ is $B$-torsionfree for each $R$-algebra $B$ which is integral over $R$;

(iii) $B \otimes T$ is $B$-torsionfree for each $R$-algebra $B$ which is torsionfree and $(n+1)$-generated as an $R$-module.

Proof: Consider a relation $r_{1} t_{1}+\ldots+r_{n} t_{n}=0$, with $r_{i} \in R, t_{i} \in T$. Put $B=R\left[r_{1} X, \ldots, r_{n} X, X^{2}, X^{3}\right] /\left(X^{2}, X^{3}\right)$. Then $B$ satisfies (i), (ii), and (iii) since

$$
B=R\left[r_{1} x, \ldots, r_{n} x\right]=R+R r_{1} x+\ldots+R r_{n} x
$$

where $r_{i} x$ denotes $r_{i} X+\left(X^{2}, X^{3}\right)$ and satisfies $\left(r_{i} x\right)^{2}=0$. In particular, $D=B \otimes T$ is $B$-torsionfree.

Put $\xi=\sum r_{i} x \otimes t_{i} \in D$. Since $r_{i} \cdot\left(r_{j} x\right)=r_{i} r_{j} X+\left(X^{2}, X^{3}\right)=r_{j} \cdot\left(r_{i} x\right)$, the canonically induced $B$-module structure on $D$ satisfies

$$
r_{j} \cdot \xi=\sum r_{j} \cdot\left(r_{i} x\right) \otimes t_{i}=\sum r_{i} \cdot\left(r_{j} x\right) \otimes t_{i}=r_{j} x \otimes \sum r_{i} t_{i}=r_{j} x \otimes 0=0 .
$$

Without loss of generality, $r_{j} \neq 0$ for some $j$. Then $r_{j}$ is a non-zerodivisor in $B$ and, since $D$ is $B$-torsionfree, we conclude $\xi=0$.

Since $B$ is generated as an $R$-module by $r_{1} x, \ldots, r_{n} x$ and 1 , an application of [3, Lemma 10, p.25] yields elements $f_{j} \in T, r_{i j} \in R$ (for $1 \leqslant i \leqslant n+1 ; 1 \leqslant j \leqslant m$ ) such that

$$
\begin{gathered}
\sum r_{i j} f_{j}=t_{i} \text { for } 1 \leqslant i \leqslant n ; \quad \sum r_{n+1, j} f_{j}=0 ; \text { and } \\
r_{1 j} r_{1} x+\ldots+r_{n j} r_{n} x+r_{n+1, j} \cdot 1=0 \in B \text { for } 1 \leqslant j \leqslant m .
\end{gathered}
$$


Since $\left\{1, X, X^{2}, \ldots\right\}$ forms a basis of the free $R$-module $R[X]$, it follows easily that $\sum r_{i j} r_{i}=0=r_{n+1, j}$ for all $j$. The assembled equations include those needed to show that $T$ is $n$-flat, and so the proof is complete.

One can regard Theorem 2.2 as a response to the question of "how much" torsionfree behaviour is needed in order to ensure $n$-flatness. Similarly, Corollary 2.3 determines "how much" $n$-flatness is needed to ensure that a finitely generated module is flat. Note that the equivalence of (1) and (5) was obtained in [2, Corollary 3.3] in case $R$ is an integral domain and $T$ is an ideal of $R$; it is interesting that only Proposition 2.1 is needed for the proof that (1) and (5) are equivalent.

Corollary 2.3. Let $R$ be a ring, $n$ a positive integer, and $T$ an $n$-generated $R$-module. Then the following conditions are equivalent:

(1) $T$ is $n$-flat over $R$;

(2) $B \otimes T$ is $B$-torsionfree for each $n$-generated $R$-algebra $B$;

(3) $B \otimes T$ is $B$-torsionfree for each $R$-algebra $B$ which is integral over $R$;

(4) $B \otimes T$ is $B$-torsionfree for each $R$-algebra $B$ which is torsionfree and $(n+1)$-generated as an $R$-module;

(5) $T$ is $R$-flat.

Proof: By earlier comments, (5) implies each of (2), (3), and (4). Moreover, by Theorem 2.2, (2), (3) and (4) each imply (1). Thus it suffices to show that (1) $\Rightarrow(5)$.

It is enough to show that the canonical homomorphism $g: I \otimes T \rightarrow T$ is a monomorphism for each ideal $I$ of $R$ (see [3, Proposition 1, p.12]). Consider $\xi \in \operatorname{ker}(g)$. Writing $T=R t_{1}+\ldots+R t_{n}$, we have $\xi=\sum r_{i} \otimes t_{i}$ for suitable $r_{i} \in I$. Put $J=\sum R r_{i}$ and $\mu=\sum r_{i} \otimes t_{i} \in J \otimes T$. Let $f$ denote the homomorphism $J \otimes T \rightarrow I \otimes T$ induced by the inclusion of $J$ in $I$. Since $f(\mu)=\xi$, we have $(g f)(\mu)=g(\xi)=0$. But $g f$ is the canonical homomorphism $J \otimes T \rightarrow T$. Thus, assuming (1), we have via Proposition 2.1 that $\mu=0$. Hence $\xi=f(0)=0$, completing the proof.

It is well known (see the first paragraph of [7]) that an integral domain $R$ is a Prüfer domain if and only if each 2-generated ideal of $R$ is $R$-flat. This is but one of several torsionfree-theoretic characterisations of Prüfer domains (some of which were referenced in the second paragraph of this paper's introduction). In the next result, we offer one more such characterisation. In view of the preceding comment, the proof of Corollary 2.4 is immediate from the proof of Corollary 2.3.

Corollary 2.4. An integral domain $R$ is a Prüfer domain if (and only if) $B \otimes I$ is $B$-torsionfree for each 2-generated ideal $I$ of $R$ and each 2-generated $R$-algebra $B$ which is torsionfree and 3-generated as an $R$-module.

We next show that "universally $n$-flat" characterises flatness. 
Corollary 2.5. Let $R$ be a ring, $T$ an $R$-module, and $n$ a positive integer. Then the following conditions are equivalent:

(1) $B \otimes T$ is $n$-flat over $B$ for each $R$-algebra $B$;

(2) $T$ is $R$-flat.

Proof: $(2) \Rightarrow(1)$ since flatness is a universal property that implies $n$-flatness. Conversely, suppose that (1) holds. Recall that $n$-flat implies 1 -flat; and 1-flat is equivalent to torsionfree (see [8, Theorem 3.3(a)]). It follows that $B \otimes T$ is $B$-torsionfree for each $R$-algebra $B$. By Theorem 2.2, $T$ is $m$-flat over $R$ for each positive integer $m$, and so $T$ is $R$-flat. (The preceding sentence can be replaced by an appeal to [12, Théorème 3.1].) The proof is complete.

REMARK 2.6. Corollary 2.5 was motivated by Lazarus's characterisation of flat as "universally torsionfree" or, equivalently, as "universally 1-flat." Another upshot of Lazarus's result is that 1 -flat is not a universal property. We close this section by recording the fact that 2 -flat is also not a universal property. Indeed, consider the example of Uda [15, Example 4.8] of an extension $R \subset T$ of integral domains where $T$ is $L C M$-stable over $R$ but not $R$-flat. By [8, Theorem 3.3(b)], $T$ is 2-flat over $R$; but, by Corollary 2.5, $T$ is not universally 2 -flat over $R$.

\section{ARE $n$-FLAT MODULES $n$-UNIVERSALLY TORSIONFREE?}

In this section, we consider the converse of Theorem 2.2. The comments in the third paragraph of the introduction serve to sharpen our focus, as follows. We assume that $R$ is an integral domain, $T$ is a torsionfree $R$-module and $n=1$; of course, $T$ is 1-flat over $R$. We ask whether $B \otimes T$ is $B$-torsionfree for each 1 -generated $R$-algebra $B$ which is torsionfree and 2-generated as an $R$-module. Proposition 3.1 records an affirmative answer in the simplest case, in which $B$ is the algebra of dual numbers over $R$.

Proposition 3.1. Let $R$ be an integral domain and $T$ a torsionfree $R$ module. Put $B=R[X] /\left(X^{2}\right)$. Then $B \otimes T$ is $B$-torsionfree.

Proof: $B=R \oplus R \delta$, where $\delta=X+\left(X^{2}\right)$ satisfies $\delta^{2}=0$. Let $D$ denote $T \oplus T$ as an abelian group, with $B$-module structure given by

$$
\left(r_{1}+r_{2} \delta\right) \cdot\left(t_{1}, t_{2}\right)=\left(r_{1} t_{1}, r_{1} t_{2}+r_{2} t_{1}\right)
$$

for $r_{i} \in R, t_{i} \in T$. It is straightforward to verify that $B \otimes T$ is isomorphic to $D$ as a $B$-module. Indeed, consider the additive homomorphism $B \otimes T \rightarrow D$ sending $\left(r_{1}+r_{2} \delta\right) \otimes t$ to $\left(r_{1} t, r_{2} t\right)$. This is a $B$-module isomorphism, with inverse given by $\left(t_{1}, t_{2}\right) \mapsto 1 \otimes t_{1}+\delta \otimes t_{2}$. Since $T$ is torsionfree over $R$ and $\left\{r_{1}+r_{2} \delta: r_{1} \neq 0\right\}$ is the set 
of non-zerodivisors of $B$, an easy calculation shows that $D$ is a torsionfree $B$-module. Hence $B \otimes T$ is torsionfree over $B$, completing the proof.

The ring $B$ in Proposition 3.1 is not an integral domain. We remove this obstacle in Proposition 3.2, and nevertheless find another case in which the riding question has an affirmative answer.

Proposition 3.2. Let $R$ be an integral domain of characteristic $\neq 2$ and let $T$ be a torsionfree $R$-module. Let $B=R+R x$ be an integral domain which contains $R$ and is not an overring of $R$. Then $B \otimes T$ is $B$-torsionfree.

Proof: Since $B$ is a ring, $x^{2}=a_{1}+a_{2} x$ for suitable $a_{1}, a_{2} \in R$. Moreover, the $a_{i}$ are uniquely determined. Indeed, the hypothesis that $B$ is not an overring of $R$, that is that $x$ does not belong to the quotient field of $R$, ensures that $B=R \oplus R x$.

If the assertion fails, then $b \cdot \xi=0$ for some nonzero elements $b \in B, \xi \in B \otimes T$. Write $b=r_{1}+r_{2} x$ and $\xi=1 \otimes t_{1}+x \otimes t_{2}$ for suitable $r_{i} \in R, t_{i} \in T$. Now

$$
0=b \cdot \xi=1 \otimes\left(r_{1} t_{1}+r_{2} a_{1} t_{2}\right)+x \otimes\left(r_{2} t_{1}+\left[r_{1}+r_{2} a_{2}\right] t_{2}\right) .
$$

By [3, Lemma 10, p.25], there exist $r_{1 j}, r_{2 j} \in R$ and $f_{j} \in T(1 \leqslant j \leqslant m)$ such that $r_{1 j}+r_{2 j} x=0$ for each $j$ and

$$
r_{1} t_{1}+r_{2} a_{1} t_{2}=\sum r_{1 j} f_{j}, \quad r_{2} t_{1}+\left[r_{1}+r_{2} a_{2}\right] t_{2}=\sum r_{2 j} f_{j} .
$$

Since $x$ is not in the quotient field of $R$, we have $r_{1 j}=r_{2 j}=0$ for each $j$. The last-displayed equations are thus a homogeneous linear system in the "unknowns" $t_{1}, t_{2}$. By Cramer's Rule, the coefficient matrix has vanishing determinant; that is, $r_{1}^{2}+r_{1} r_{2} a_{2}-r_{2}^{2} a_{1}=0$. Hence, $y=r_{1} / r_{2}$ is a root of the polynomial $X^{2}+a_{2} X-a_{1}$. By the quadratic formula,

$$
y=\left[-a_{2} \pm\left(a_{2}^{2}+4 a_{1}\right)^{1 / 2}\right] / 2 .
$$

Also by the quadratic formula,

$$
x=\left[a_{2} \pm\left(a_{2}^{2}+4 a_{1}\right)^{1 / 2}\right] / 2 .
$$

Thus, $2 y+a_{2}= \pm\left(2 x-a_{2}\right)$. It follows that $x$ is in the quotient field of $R$, the desired contradiction. The proof is complete.

REMARK 3.3. (a) Many of the proofs in this paper use the criterion of Bourbaki [3, Lemma 10, p.25] for a tensor to vanish. It is possible to fashion an alternate proof of Proposition 3.1 along these lines. Moreover, by using standard tensor product techniques, one can discover the module structure and isomorphisms developed in the earlier proof of Proposition 3.1. We leave the details to the interested reader. 
(b) In the above vein, one could ask whether $B \otimes T$ is $B$-torsionfree when $T$ is an overring of an integral domain $R$ and $B$ is an $R$-algebra. Since tensor product commutes with direct limit, $T$ may be assumed of finite type over $R$. Consider the simplest case, $T=R[1 / r]=R_{r}$ for some nonzero $r \in R$. Then $B \otimes T \cong B_{r}$. An easy calculation shows that if $b$ is a non-zerodivisor in $B$, then $b / 1$ is a non-zerodivisor in $B_{r}$. Hence, $B \otimes T$ is $B$-torsionfree.

It is time for a final sharpening of focus. Since Proposition 3.2 did not produce the anticipated counterexample, let us take $B$ to be an overring of $R$. What should the "decisive" $T$ be? It should be, in some sense, "well-connected" to both $R$ and $B$. An obvious candidate from this point of view is to take $R$ to be a pseudo-valuation domain $(P V D)$, in the sense of [11]. In other words, $R$ will be a quasilocal integral domain having the same maximal ideal, $M$, as some valuation overring $V$; and $T$ will be taken to be $M$. In any counterexample, $T=M$ is not $R$-flat. For a $P V D$, this is characterised by $M^{2} \neq M[6$, Theorem 2.3(a)]; this condition holds, by Nakayama's Lemma, if $V$ is Noetherian, that is, a $D V R$. Since $B$ is generated by an element satisfying a quadratic equation over $R$, it is natural to arrange $B=R[i]$, where $i^{2}=$ -1 . With these comments, the plausible shape of the most natural counterexample is now clear. It is presented as our final result.

EXAMPLE 3.4: Consider the Noetherian integral domain $R=\mathbf{R}+X \mathrm{C}[[X]]$, its overring $B=C[[X]]$, and their common maximal ideal $T=X B$. Of course, $T$ is a torsionfree $R$-module; and $B=R[i]=R+R i$ is a 1-generated $R$-algebra which is torsionfree and 2-generated as an $R$-module. Moreover, $B \otimes T$ is not $B$-torsionfree.

Proof: Consider $b=i X \in B$ and $\xi=1 \otimes X+i \otimes i X \in B \otimes T$. Then

$$
\begin{aligned}
b \cdot \xi & =i X \otimes X+i^{2} X \otimes i X=(i X) \cdot 1 \otimes X-1 \otimes i X \\
& =1 \otimes(i X) X-1 \otimes X(i X)=0 .
\end{aligned}
$$

It suffices to show that $\xi \neq 0$. Suppose not. Since $T=R X+R i X$, an appeal to [3, Lemma $10 \mathrm{p}$.25] yields elements $r_{1 j}, r_{2 j} \in R$ and $f_{j} \in T(1 \leqslant j \leqslant m)$ such that

$$
r_{1 j} X+r_{2 j} i X=0, \quad 1=\sum r_{1 j} f_{j}, \text { and } i=\sum r_{2 j} f_{j} .
$$

There are now several ways to derive a contradiction. Here is perhaps the fastest. The second (respectively, third) of the displayed equations ensures that 1 (respectively, $i$ ) lies in (the $R$-module) $T$, the desired contradiction. The proof is complete.

\section{REFERENCES}

[1] T. Akiba, $L C M$-stableness, $Q$-stableness and flatness, Kobe J. Math. 2 (1985), 67-70. 
[2] D.D. Anderson and D.E. Dobbs, 'Flatness, LCM-stability, and related module-theoretic properties', J. Algebra 112 (1988), 139-150.

[3] N. Bourbaki, Commutative Algebra (Addison-Wesley, Reading, 1972).

[4] S.U. Chase, 'Direct products of modules', Trans. Amer. Math. Soc. 97 (1960), 457-473.

[5] E.D. Davis, 'A remark on Prüfer rings', Proc. Amer. Math. Soc. 20 (1969), 235-237.

[6] D.E. Dobbs, 'On the weak global dimension of pseudo-valuation domains', Canad. Math. Bull. 21 (1978), 159-164.

[7] D.E. Dobbs, 'On flat finitely generated ideals', Bull. Austral. Math. Soc. 21 (1980), 131-135.

[8] D.E. Dobbs, 'On the criteria of D.D. Anderson for invertible and flat ideals', Canad. Math. Bull. 29 (1986), 25-32.

[9] R. Gilmer, Finite element factorization in group rings: Lecture Notes in Pure and Appl. Math. 7 (Dekker, New York, 1974).

[10] A. Hattori, 'On Prüfer rings', J. Math. Soc. Japan 9 (1957), 381-385.

[11] J.R. Hedstrom and E.G. Houston, 'Pseudo-valuation domains', Pacific J. Math. 75 (1978), 137-147.

[12] M. Lazarus, 'Fermeture intégrale et changement de base', Ann. Fac. Sci. Toulouse Math. 6 (1984), 103-120.

[13] F. Richman, 'Generalized quotient rings', Proc. Amer. Math. Soc. 16 (1965), 794-799.

[14] J. Sato and K. Yoshida, 'The LCM-stability on polynomial extensions', Math. Rep. Toyama Univ. 10 (1987), 75-84.

[15] H. Uda, ' $L C M$-stableness in ring extensions', Hiroshima Math. J. 13 (1983), 357-377.

[16] H. Uda, ' $G_{2}$-stableness and $L C M$-stableness', Hiroshima Math. J. 18 (1988), 47-52.

Department of Mathematics

University of Tennessee

Knoxville, Tennessee 37996-1300

United States of America 